\title{
Ana J. García Sáez
}

Analyzing super-res microscopy images ASAP, strengthened by Ultimate Frisbee.

S ometimes you just have to build what you need, and you do so with others because you like team sports such as handball, basketball and Ultimate Frisbee. Such is the case for biologist and biophysicist Ana J. García Sáez from University of Tübingen's Interfaculty Institute of Biochemistry. She studies cellular machineries with super-resolution microscopy, mainly stochastic optical reconstruction microscopy (STORM).

García Sáez wants to understand cell death. When a cell is about to commit suicide, as a result of external factors or as part of its life cycle, holes form in the mitochondrial membrane through which the organelle's innards will ooze into the cytosol. "They don't come back from that," she says. "I want to know the process." She wants to be quantitative and automated when collecting this knowledge from super-resolved structures decorated with fluorophores. But the available software tools were either not a good fit or quite pricey.

She and her postdoctoral fellow John Danial, now a fellow at the University of Cambridge, built a software tool to automate extraction of information: it's called Automated Structures Analysis Program, or ASAP, available here and as part of their new paper. When they tested the first versions in the lab, "it made everyone so happy," she says. What used to take a week could now be accomplished in a day. Everyone in her lab and others, too, offered feedback, and Danial tweaked the code. "He is the programmer, not me," says García Sáez. At its core, ASAP applies machine learning, letting users choose from several different classifiers, such as a support vector machine approach.

The team trained the system to extract, identify and classify detected structures, which can be lines, rings or arcs and which are statistically defined. A ring is the telltale sign of a hole in the mitochondrial membrane. They trained the tool with manually annotated images and the team validated ASAP on various cellular structures, including nuclear pores. That was the slow part of the development, says García Sáez. The invested time accelerates work in her lab, and she hopes it will do so for other labs, too. Each experimental image

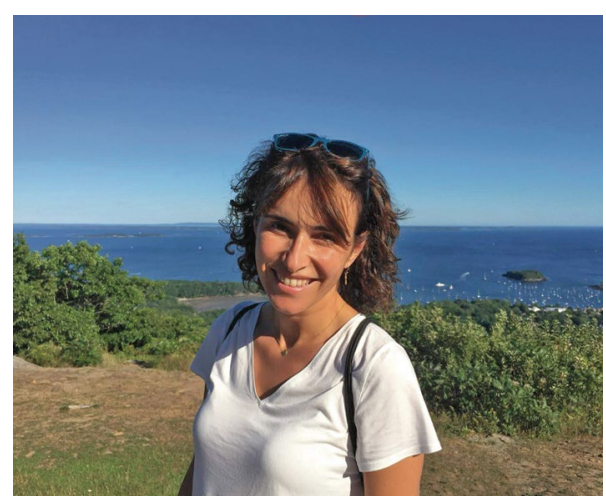

to immediately go back to the lab and test what we just discovered," says Walczak. She always thinks of ways to apply the techniques she masters to his scientific questions. He has always enjoyed mentoring García Sáez, which happened from afar since they didn't work at the same institution. When he saw an opportunity, "I pointed it out to her and convinced her she should go for it." Beyond "a wonderful sense of humor and lovely personality," he says, she is a great salsa dancer, "which is always a plus in life," especially for biophysicists.

When García Sáez is not in the lab, she Ana J. García Sáez. Credit: A. Villunger

can contain hundreds of objects to identify, she says. That's hard to do consistently by eye. Then there is "human cognitive bias" to contend with. "People see things differently," she says. "It's normal." One person sees the shape of fluorescing tags as an arc while another person calls it a ring, and vice versa. These differing classifications can skew data analysis. "I wanted to address this bias," she says. Several people trained the tool, and annotation was double-blinded, also to address, she jests, too much willingness to detect certain structures.

García Sáez takes her fondness for team sports into the lab: ASAP was developed collaboratively. Even though Danial is now in London, they plan to continue to advance the tool together. Her ideas germinate most anywhere, she says: in the lab, at conferences, when with her children, on a run or a hike. "I mainly get ideas in conversations with others," she says. She seeks out discussions in her lab and with colleagues elsewhere.

Her mentor and collaborator Henning Walczak, from University College London, says that García Sáez struck him as one of the few scientists who combine brilliant thinking "with a natural curiosity and enthusiasm for understanding the unexplored." It's a combination that positions her well to best use physics for understanding biology. "I cannot think of a single conversation I've had with her that would have left me without the urge spends time with family. She has two preschool-age, trilingual children-they speak Spanish, English and German. She squeezes in a game of Ultimate Frisbee when she can and plays in a team of men and women. "I like the intense workout of the game," she says.

\section{"I mainly get ideas in conversations with others."}

García Sáez grew up Buñol, a village near València, Spain, and gladly takes part in the tomatina street festival, in which all are doused with tomatoes. She attended the University of València. During her PhD research in molecular biology she did a fellowship at the Technical University of Dresden, and she returned to Dresden as a postdoctoral fellow. She held a double appointment at the German Cancer Research Center and the Max Planck Institute for Intelligent Systems and has now made Tübingen her home. She misses her native Spain's mild weather and sun, but it's a quick trip from Germany. She and family visit a few times a year, and she enjoys the fray of the verbena festivals with bands and street dancing.

Vivien Marx

Published online: 28 June 2019 https://doi.org/10.1038/s41592-019-0463-2

Reference analysis of super-resolved structures using ASAP. Nat. Methods https://doi.org/10.1038/s41592-0190472-1 (2019).
Danial, J. S. H. \& Garcia-Saez, A.-J. Quantitative 